Cahiers $d u$ MONDE RUSSE

\section{Cahiers du monde russe}

Russie - Empire russe - Union soviétique et États indépendants

$50 / 4 \mid 2009$

Varia

\title{
Donald Filtzer, Wendy Z. Goldman, Gijs Kessler, Simon Pirani, eds., A Dream Deferred
}

Jean-Paul Depretto

\section{OpenEdition}

Journals

Édition électronique

URL : https://journals.openedition.org/monderusse/7237

DOI : $10.4000 /$ monderusse. 7237

ISSN : $1777-5388$

Éditeur

Éditions de l'EHESS

\section{Édition imprimée}

Date de publication : 15 décembre 2009

Pagination : 915-920

ISBN : 978-2-7132-2261-0

ISSN : $1252-6576$

\section{Référence électronique}

Jean-Paul Depretto, « Donald Filtzer, Wendy Z. Goldman, Gijs Kessler, Simon Pirani, eds., A Dream Deferred », Cahiers du monde russe [En ligne], 50/4 | 2009, mis en ligne le 12 janvier 2011, consulté le 03 septembre 2022. URL : http://journals.openedition.org/monderusse/7237 ; DOI : https://doi.org/ 10.4000/monderusse. 7237

Ce document a été généré automatiquement le 3 septembre 2022.

Tous droits réservés 


\title{
Donald Filtzer, Wendy Z. Goldman, Gijs Kessler, Simon Pirani, eds., A Dream Deferred
}

\author{
Jean-Paul Depretto
}

\section{RÉFÉRENCE}

Donald FILTZER, Wendy Z. GOLDMAN, Gijs KESSLER, Simon PIRANI, eds., A Dream Deferred.

New Studies in Russian and Soviet Labour History. Berne : Peter Lang, 2008, 508 p.

1 Ce livre collectif est issu de la conférence «Labour History of Russia and the Soviet Union: Work in Progress ", tenue en mars-avril 2005 à l'Institut international d'histoire sociale d'Amsterdam. Il rassemble une sélection de communications remaniées. Notons qu'un important effort éditorial rend l'ouvrage accessible à un public plus large que celui des seuls spécialistes de la Russie/URSS, avec en annexes cinq cartes, une bibliographie générale, un glossaire et trois index (thèmes, noms, toponymes).

2 La notion de « labour » ici est entendue dans un sens très large : elle englobe paysans et ouvriers, à la ville et à la campagne, à l'usine et aux champs comme à la maison. La matière a été regroupée en trois grandes parties d'importance inégale: ouvriers et politique ouvrière (1880-1941) ; travailleurs et travail : coercition et stimulants ; famille, nourriture et travail : stratégies de survie, de 1884 à aujourd'hui.

Le livre s'ouvre sur l'article de Nikolai V. Mikhailov consacré aux « organisations ouvrières non partisanes à Saint-Pétersbourg et en province avant et pendant la première révolution russe ". L'auteur soutient que les expériences passées des ouvriers dans le mir ont fourni un modèle pour les organisations ouvrières des usines. Les sociétés d'entraide mises en place à l'initiative des patrons forment une autre source d'expérience de l'organisation collective: les ouvriers y prennent l'habitude de participer à des réunions, tenir des élections et payer des cotisations. Ils ont une très claire conscience de classe et n'acceptent pas employés et employeurs dans les syndicats; ils développent 
leurs propres idées, distinctes de celles que leur attribuent les couches éduquées de la société.

4 Christine Ruane étudie la grève qui a paralysé les entreprises de l'habillement à Moscou pendant plus d'un mois à l'automne 1906. Ce sujet a été délaissé par les historiens qui ont en général préféré s'intéresser aux usines et négligé les groupes artisanaux. À cette époque, les compagnons de la confection voient leur métier et leur mode de vie disparaître sous leurs yeux : cette perte de statut les radicalise et les conduit à s'opposer à leurs employeurs, ainsi qu'aux ouvriers prêts à travailler " pour l'ennemi ». Cette grève montre que les ouvriers aussi ont joué un rôle dans la déstabilisation de la Russie, qui se poursuit après la révolte de décembre 1905 ; leurs protestations auraient contribué à la décision de Stolypin qui aboutit au coup d'État de juin 1907.

Sarah Badcock examine l'identité et l'expérience ouvrières dans les usines de Sormovo (banlieue de Nižnij Novgorod) en 1917. Le mouvement révolutionnaire y est sensiblement différent de ce que les historiens ont pu observer à Petrograd et à Moscou et caractérisé par la fragmentation, la compétition et la confusion. S. Badcock conclut qu'il y a peu d'unité entre ouvriers et soldats, que les autorités locales réussissent à opposer. Par ailleurs, les ouvriers de Sormovo sont mieux approvisionnés que les travailleurs de Nižnij Novgorod, privilège qui suscite de l'hostilité à leur égard. L'atmosphère très politisée de Sormovo est plutôt une exception dans la région où, en dehors des grandes agglomérations ouvrières, la politique des partis a un impact très limité. Enfin, ces ouvriers de banlieue s'identifient durablement aux socialistes révolutionnaires, malgré la montée de l'influence bolchevique.

Simon Pirani ${ }^{1}$ tente de montrer comment le parti bolchevik restructure ses rapports politiques avec la classe ouvrière après la guerre civile. De 1921 à 1923, cette relation commence sous forme d'un contrat social : les ouvriers améliorent la productivité du travail et cèdent le pouvoir de décision au parti communiste qui, en retour, assure une amélioration régulière des conditions de vie. Ce contrat implique le développement de techniques de mobilisation de masse, appelée à se substituer à la démocratie participative : les ouvriers sont encouragés à manifester en public leur soutien au nouvel ordre. Pirani prend pour exemple Moscou en 1922 pour mettre en évidence ce processus, à travers deux campagnes : la confiscation des objets précieux des églises et le procès des socialistes révolutionnaires. Les ouvriers soutiennent la première, mais passivement, tandis que des centaines de milliers d'entre eux manifestent leur appui à la seconde. Pirani en déduit que ni la répression ni la coercition ne fournissent une explication suffisante: le niveau de vie ayant nettement progressé, cela signifie que l'État avait honoré son contrat.

7 En 1921, le Xe Congrès du parti bolchevik interdit les factions et blâme l'opposition ouvrière. En 1926, deux des leaders de cette opposition, A. Šljapnikov et S. Medvedev, font l'objet d'enquêtes de la Commission centrale de contrôle dans le cadre de « l'affaire de Bakou ». Barbara Allen, auteur d'une thèse sur A. Šljapnikov², montre comment la définition de "l'activité fractionnelle» évolue dans le temps, s'élargissant peu à peu pour inclure des conversations et des réunions entre communistes qui s'opposent au déficit croissant de démocratie dans le parti communiste et à la perte d'influence des ouvriers. La Commission centrale de contrôle aurait ainsi contribué à installer la scène des procès politiques des années 1930.

Les rapports entre l'opposition communiste de gauche (trotskistes, "décistes ») et la classe ouvrière en 1928-1929 font l'objet de l'article d'Aleksei Gusev. Selon l'auteur, les 
ouvriers répondent positivement aux programmes d'opposition, mais la construction d'un mouvement politique alternatif échoue pour deux raisons: d'une part, les «sommets» de l'opposition ne peuvent rompre complètement avec la bureaucratie gouvernante et continuent à croire en une possible réforme interne du parti communiste; d'autre part, la jeune génération ouvrière n'a pas d'illusions sur l'« État ouvrier ", mais la répression fait vite son œuvre et, en 1929, la plupart des groupes clandestins sont liquidés.

9 La publication en cours des rapports de l'OGPU à Stalin ${ }^{3}$ et des données sur l'entreprise métallurgique Serp i Molot permettent à Kevin Murphy de retracer l'évolution des grèves de 1922 à 1932. L'auteur distingue quatre étapes : au début de la NEP, la grève est un phénomène répandu et l'État recourt en général à des accommodements plutôt qu'à la répression; un "contrat social " négocié en 1925 permet de prévenir les grèves avec succès; à la fin de la NEP, ce compromis a été peu à peu érodé par les campagnes sur la productivité et la réduction des coûts; enfin, les années 1928-1932 voient le déclin des grèves, qui ont pris en revanche une plus grande signification politique.

10 Wendy Z. Goldman expose les conclusions de son livre sur la Terreur à l'usine ${ }^{4}$. Elle analyse la relation entre les directives centrales du parti communiste et les réponses à ces directives dans les entreprises, en se concentrant sur les comités de parti et les ouvriers de Moscou. Au début, les communistes et les ouvriers, peu disposés à découvrir des « ennemis masqués » dans leurs propres rangs, résistent aux efforts du parti pour les impliquer dans la répression. Mais à l'automne 1936, les leaders soviétiques lancent une nouvelle campagne contre le « sabotage " qui réussit à étendre la chasse aux ennemis à tous les lieux de travail. Au printemps 1937, les comités de parti passent le plus clair de leur temps à examiner dénonciations et enquêtes; de leur côté, les ouvriers accusent directeurs, ingénieurs et fonctionnaires locaux d'abus.

11 La seconde partie du volume s'ouvre sur un article de Lynne Viola consacré aux colons spéciaux déportés dans le nord de la Russie d'Europe au début des années 1930. Cette main-d'œuvre n'est ni efficace ni, paradoxalement, bon marché. Les plans grandioses de Moscou heurtent de front les réalités locales : sur place prévalent les « conditions de la taïga", et non le "pouvoir soviétique». Les conceptions officielles de la lutte des classes, qui justifiaient les abus du personnel d'encadrement, aggravaient la situation des paysans dékoulakisés. Au final, il apparaitt que les peuplements spéciaux ont donné lieu aux pires atrocités dans l'exploitation de la main-d'œuvre captive.

12 Ces conclusions de Lynne Viola rejoignent la description par Jean Lévesque des problèmes de gestion rencontrés par les présidents de kolkhozes qui ne parviennent pas à atteindre les buts planifiés par l'État (1932-1953). Les kolkhozes sont mal gouvernés pendant la plus grande partie de la période stalinienne et leurs présidents ne sont pas à la hauteur des attentes de Moscou. Restant trop peu de temps en poste, connaissant mal la dynamique interne des villages et très vulnérables à la pression de l'administration des districts, ces derniers ne constituent pas un fondement solide du système. Au total, le kolkhoze apparaît comme un maillon faible de l'État stalinien.

13 C'est un aspect plus souriant du régime qu'aborde Diane P. Koenker : le tourisme ouvrier dans les années 1930. Alors que voyages, maisons de repos et vacances n'étaient auparavant accessibles qu'aux riches, les dirigeants soviétiques cherchent en principe à les rendre accessibles à la plupart des ouvriers. En pratique, seule une minorité d'entre eux peut pratiquer le tourisme dans l'entre-deux-guerres, les maisons de repos syndicales n'accueillant que les ouvriers des entreprises les plus favorisées. Mais l'espoir 
de bénéficier de ces avantages fonctionne comme un stimulant : les vacances sont une promesse et certains travailleurs méritants peuvent malgré tout accéder à ces conquêtes du socialisme.

Barbara Alpern Engel a exploité deux cents dossiers des archives de la Chancellerie impériale pour la réception des requêtes. Il y est question de femmes mariées, appartenant aux couches populaires, qui, à la fin de la période tsariste, demandent un passeport pour vivre séparées de leur époux. L'auteur explore la signification du travail dans la vie de ces femmes dont le mariage a échoué: il ne s'agit pas seulement d'ouvrières d'usine, mais aussi de domestiques, de journalières, d'artisanes, etc. Elle met l'accent sur deux conclusions. En Russie, le travail extradomestique des femmes est largement moins problématique, même pour l'élite, que dans de nombreux pays d'Europe de l'Ouest ou aux États-Unis. Par ailleurs, dans certains cas au moins, l'emploi rémunéré des femmes encourage leur indépendance en les poussant à résister à la négligence ou aux abus du mari. Enfin il permet à des femmes mariées de reconstruire leur vie.

Gijs Kessler analyse comment les ménages urbains affrontent la série de chocs démographiques et économiques des années 1917-1941 : ils peuvent alléger les effets du chômage dans les années 1920 et de la baisse du salaire réel pendant le premier plan quinquennal en mettant en commun les revenus. Leur configuration est fortement remodelée par les pertes en hommes de la longue période de guerre 1914-1921. La répression des années 1930 et la Seconde Guerre mondiale accentuent le déséquilibre entre hommes et femmes, devenu un trait durable de la vie russe au $\mathrm{xx}^{\mathrm{e}}$ siècle. Les femmes âgées ont du mal à se remarier; elles s'occupent des petits-enfants, permettant ainsi à la génération intermédiaire de travailler. Les salariées subissent une double journée de travail, au détriment du temps de repos, de loisirs ou d'éducation.

16 Donald Filtzer a réalisé un impressionnant travail d'archives. À partir notamment des enquêtes locales sur les budgets des ménages menées par la Direction centrale des statistiques, il explore l'impact de la famine de 1947 dans les régions industrielles de RSFSR qui n'ont pas été occupées pendant la Seconde Guerre mondiale. On sait que cette famine a surtout touché le sud de l'Ukraine et la Moldavie, mais elle a affecté profondément ouvriers et paysans dans toute l'URSS. Ce sont les familles d'ouvriers qui se révèlent les plus vulnérables. Contrairement à ce qui s'était passé en 1932-1933, la mortalité infantile est plus élevée dans les villes qu’à la campagne. Les paysans compensent les effets de la famine grâce à leur plus grande facilité d'accès au lait et aux pommes de terre. L'analyse des enquêtes de budgets permet d'établir qu'au-delà de la famine de 1947, les ouvriers soviétiques et leurs familles continuent à recevoir une nourriture inférieure aux normes jusque dans les années 1950:2 686 calories par personne en 1955, soit un chiffre inférieur de plus de 200 calories à la ration paysanne moyenne.

17 Le travail dans l'agriculture est normalement une activité de campagnards, constate Andrei Markevich, mais vers 1950 ce n'est pas le cas pour les villes soviétiques, où beaucoup de citadins s'adonnent à une agriculture subsidiaire pour se procurer un revenu supplémentaire. Cette pratique de survie, étudiée ici sur la période 1941-1964, montre clairement combien le niveau de vie est bas en URSS à cette époque. Sa contribution à l'alimentation de la population soviétique pendant la guerre et à la victoire sur l'Allemagne nazie ne saurait être sous-estimée. Dans les années 1950, cette pratique continue: elle permet aux citoyens soviétiques de faire face aux pénuries dans le commerce d'État et d'économiser de l'argent pour acheter des biens de consommation. 
L'agriculture subsidiaire contribue ainsi largement à la renaissance du secteur privé à la fin de la guerre et dans les premières années de l'après-guerre. Hruščev finit par s'y attaquer, ce qui contribue à l'explosion de Novočerkassk en 1962. Sous BreÂnev, l'agriculture subsidiaire ne retrouvera jamais son niveau des années 1940 et 1950; désormais réservée aux pauvres, elle est relayée à la fin de la période soviétique par le phénomène de la datcha.

Le volume se clôt sur la période récente, avec l'article de Sergei A. Afontsev, intitulé « Le choix d'activités rémunératrices. Les ménages urbains russes et les défis de la transition ». L'enquête permet d'identifier trois stratégies majeures de ces ménages : la diversification des revenus, le remplacement de biens achetés par des biens produits à la maison et la spécialisation du travail par sexe et par âge. La première stratégie est une tentative d'échapper à la pauvreté et d'atteindre un niveau de revenu " satisfaisant »; les contraintes financières semblent être la principale motivation pour adopter la deuxième. Mais, depuis la fin des années 1990, la hausse du niveau de vie cause d'importants changements dans le comportement des ménages: l'agriculture subsidiaire et les emplois secondaires mal payés auraient ainsi de bonnes chances de disparaitre.

Bref, ce livre, où chacun puisera en fonction de ses centres d'intérêt, mérite l'appréciation la plus élogieuse: il restera comme un ouvrage de référence, dû aux meilleurs spécialistes du domaine. Écrit par des chercheurs de cinq pays, il fait le point sur l'état de notre connaissance du monde du travail russe et soviétique. C'est donc un guide précieux qui offre une très riche information. Les vingt pages de la bibliographie collective rendront notamment de grands services.

\section{NOTES}

1. Voir l'ouvrage récent de Simon Pirani, The Russian Revolution in Retreat, 1920-1924: Soviet Workers and the New Communist Elite, Londres-New York : Routledge, 2008.

2. Thèse intitulée : "Worker, Trade Unionist, Revolutionary ", et soutenue à l'université de l'Indiana en 2001.

3. "Soveršenno sekretno » : Lubianka-Stalinu o položenii v strane (1922-1934 gg.), 7 vol., M., 2002-2004.

4. Wendy Z. Goldman, Terror and Democracy in the Age of Stalin: The Social Dynamics of Repression, Cambridge : Cambridge University Press, 2007 (cf. mon compte rendu in Revue des Études slaves, 80 (4), 2009, p. 490-493). 